\section{Remarkable Optical Properties of the Alkali Metals}

WooD $^{1}$ has shown that the alkali metals become highly transparent in the ultra-violet region, there being for each metal a definite wave-length above which the metal becomes opaque. Kronig ${ }^{2}$ has used the quantum theory of the optical properties of metals previously developed by $\mathrm{him}^{3}$ to account qualitatively for the phenomenon, and has suggested that it is due to the absorption bands caused by the periodicity of the lattice. Hill ${ }^{4}$ has made a similar suggestion. The purpose of the present note is to show that if the electrons are treated as free (moving in no field), we can not only account qualitatively for the phenomenon observed by Wood, but also can predict correctly the approximate values of the critical wave-length. We may deduce that the influence of the absorption bands is small.

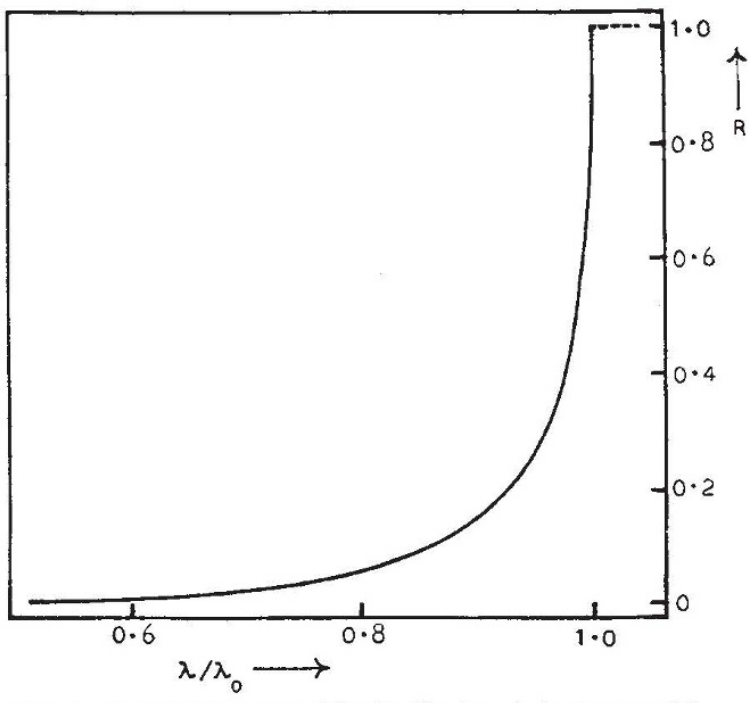

FIa. 1. Reflectivity of a metal using the free electron gas model.

Kronig has shown that, for free electrons, quantum and classical theories give the same result; and, moreover, that for the frequencies in question the resistance of the metal can have little influence, since the time between collisions of the electrons with the lattice (greater than $10^{-14}$ sec.) is greater than the period of oscillation of the electric field. $\mathrm{We}_{\theta}$ thus have for the polarisation $P$ of the electron gas :

$$
P=-\left(N e^{2} / 4 \pi^{2} m v^{2}\right) E,
$$

where $N$ is the number of electrons (or atoms for the alkalis) per unit volume, $v$ is the frequency of the light, and $E$ is the effective field (not $E+4 \pi / 3 P$, as in the theory of the polarisation of a dielectric, cf. Tonks, NAture, July 15 and Nov. 4, 1933, pp. 101, 710 , or Norton, NATURE, Oct. 28,1933 , p. 676 , who discuss the polarisation of the Heaviside layer). The dielectric constant $\varepsilon$ is then

$$
\varepsilon=1-N e^{2} / \pi m \nu^{2}
$$

There is a critical frequency $\nu_{0}$ or wave-length $\lambda_{0}$ when $\varepsilon$ is zero, that is, at

$$
\nu_{0}^{2}=\left(c / \lambda_{0}\right)^{2}=N e^{2} / \pi m
$$

When $\lambda>\lambda_{0}$, light is totally reflected, and when $\lambda<\lambda_{0}$, the proportion $R$ of light reflected is

$$
R=\left(\frac{n-1}{n+1}\right)^{2}
$$

where $n$, the refractive index, is equal to $\varepsilon^{1 / 2}$. The dependence of $R$ upon $\lambda$ is shown in Fig. 1, and has the general characteristics found by Wood.

The point $\lambda_{0}$ in the spectrum at which transparency commences is given by Wood as follows: Cs, 4400 A. ; Rb, 3600 A. ; K, 3150 A. ; Na, 2100 A. ; $\mathrm{Li}, 2050 \mathrm{~A}$. Equation (1) gives for $\lambda_{0}$ the following values : Cs, $3600 \mathrm{~A}$. ; Rb, $3200 \mathrm{~A}$. ; K, $2900 \mathrm{~A}$. ; $\mathrm{Na}, 2100 \mathrm{~A}$; Li, $1550 \mathrm{~A}$.

\section{H. H. Wills Physics Laboratory,}

Clarence Zener.

\section{Bristol.}

Nov. 11

1 Wood, Nature, 131, 582, April 22, 1933. Phys. Rev., 44, 353; 1933.

2 Kronig, NATURe, 132, 601, Oct. 14, 1933.

3 Kronig, Proc. Roy. Soc., A, 124, 409; 1929; 133, 255; 1931

4 Hill, Rev. Sci. Inst., 4, 525; 1933.

\section{Raman Spectrum of Fluorobenzene}

BRADLEY ${ }^{1}$ has studied the Raman spectra of $\mathrm{CHFCl}_{2}$ and $\mathrm{CF}_{2} \mathrm{Cl}_{2}$, which appear to be the only fluorine compounds so far investigated. I have recently studied mono-fluorobenzene, and its Raman frequencies are found to be (in $\mathrm{cm}^{-1}$ ) :

$$
\begin{aligned}
\nu_{\mathrm{R}}= & 243(5), 424(1), \quad 521(3), \quad 617(3), \quad 757(2), \\
& 808(5), 1012(7), 1065(1), 1157(4), 1220(4), \\
& 1275(1), 1413(1), 1600(5), 3074(7) .
\end{aligned}
$$

The numbers within brackets indicate the approximate intensities in arbitrary units. The prominent frequency of $1220 \mathrm{~cm}^{-1}$ seems to be characteristic of the aromatic $\mathrm{C}-\mathrm{F}$ binding. This value agrees well with $\nu_{\mathrm{R}}=1236 \mathrm{~cm} .^{-1}$ calculated, in the wellknown manner, from the corresponding heat of dissociation.

Another characteristic feature of the spectrum is the large shift of the two prominent benzene frequencies 991 and 3063, produced by the fluorine substitution ; indeed, the shifts are the largest so far noticed for these frequencies in benzene derivatives.

210, Bowbazar Street, N. Gopala Pai. Caleutta.

Nov. 12.

${ }^{2}$ Phys. Rev., 40, 908; 1932.

Molecular Orientations in p-Diphenylbenzene Crystal

IN recent papers ${ }^{1}$ we have shown how a correlation of the principal diamagnetic susceptibilities of an organic crystal with those for the molecules of the substance, offers a convenient method for determining the orientations of the molecules in the crystal lattice. The crystal of $p$-diphenylbenzene, the

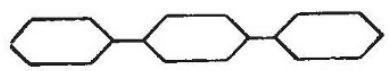

structure of which has recently been analysed by X-ray methods by Miss Pickett ${ }^{2}$, is a very suitable substance for determination of molecular orientations by this method. The crystal belongs to the monoclinic system and is found by Miss Pickett to contain two molecules in the unit cell. The molecule has a centre of symmetry; the three benzene rings lie in a plane, and their centres are in a line. 